\title{
Aspirin inhibits growth of ovarian cancer by upregulating caspase-3 and downregulating bcl-2
}

\author{
LIN LI $^{1 *}$, XIAOGANG MAO ${ }^{1 *}$, XIAOMIN QIN $^{1}$, MIN ZHOU $^{1}$, HUI XING $^{1}$, \\ FAN DONG $^{2}$, XIAOYUAN JIANG ${ }^{3}$ and WENHUI ZHUANG ${ }^{4}$
}

\begin{abstract}
${ }^{1}$ Department of Gynaecology and Obstetrics, Xiangyan Central Hospital, Hubei University of Arts and Science, Xiangyang, Hubei 441021; ${ }^{2}$ Zhongshan Hospital, Fudan University, Shanghai 200032; ${ }^{3}$ Dongfang Hospital,

The Second Clinical Medical College of Beijing University of Chinese Medicine (BUCM),

Beijing 100078; ${ }^{4}$ School of Life Sciences, Fudan Univesity, Shanghai 200433, PR. China
\end{abstract}

\author{
Received December 28, 2015; Accepted May 18, 2016
}

\begin{abstract}
The aim of the present study was to investigate the effect and mechanism of different concentrations of aspirin in inhibiting the ovarian cancer of p53N236S gene knockin mice. In total, 28 male p53S mice, with an age range of 4-6 weeks and weight of 20-25 g were selected. The animals were transplanted with SKOV3 cells to establish subdermal human ovarian cancer. The mice were randomly divided into different groups according to the aspirin concentrations (mmol/l) used, i.e., $0,1,2$ and 3. Subsequently, intraperitonea injection was performed once every two days for 3 weeks. The tumor volume, lifetime, tumor cell proliferation inhibition rates, caspase- 3 protein and bcl- 2 protein expression of the four groups were analyzed and compared. Following aspirin treatment for 1, 2 and 3 weeks, the tumor volume of the $3 \mathrm{mmol} / \mathrm{l}$ aspirin group was significantly smaller than the other groups $(\mathrm{P}<0.05)$. The higher concentration of aspirin led to a smaller tumor size $(\mathrm{P}<0.05)$. The cell proliferation inhibition rate of the $3 \mathrm{mmol} / 1$ aspirin group was significantly larger than that of other groups $(\mathrm{P}<0.05)$. The relative expression level of caspase- 3 , bcl-2 protein of the $3 \mathrm{mmol} / \mathrm{l}$ aspirin group was significantly improved and reduced, respectively. In conclusion, aspirin can inhibit the growth of ovarian cancer of p53S rats due to its upregulation of the expression of caspase-3 protein and downregulation of the expression of bcl-2 protein.
\end{abstract}

Correspondence to: Dr Hui Xing, Department of Gynaecology and Obstetrics, Xiangyan Central Hospital, Hubei University of Arts and Science, 136 Jinzhou Road, Xiangyang, Hubei, 441021, P.R. China

E-mail: xing_hui1212@163.com

* Contributed equally

Key words: aspirin, p53S mice, ovarian cancer, caspase-3 protein, bcl-2 protein

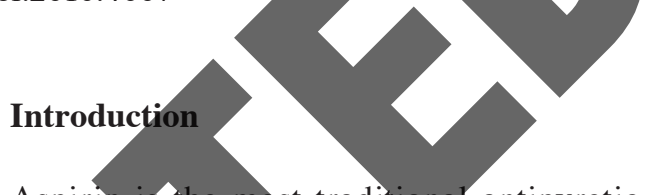

Aspirin is the most traditional antipyretic, analgesic, and anti-platelet drug. Aspirin is effective for obstetrical and gynecological diseases, such as pregnancy-induced hypertension, fetal growth restriction, anti-phospholipid syndrome, assisted reproductive technology, endometriosis, as well as ovarian cancer, endometrial and cervical cancer (1). However, the specific pharmacological mechanism, safety and adverse reactions remain to be investigated. The p53 gene is an important tumor suppressor, although the tumor-promoting effect of p53 knockout mice is not considered optimal (2).

Loss of regulation of tumor cells led to tumor occurrence and the monitoring of the mechanisms involved in DNA damage, such as cell senescence and apoptosis (3). Thus, tumor cells had to reactivate the telomere to prolong the mechanism. Approximately $85-90 \%$ tumors occurred by reactivating the telomerase. Telomerase reactivation may escape the inhibition of senescence, promoting the formation of tumor cells whose proliferation was not regulated by the body (4). A further 10-15\% tumor maintained the length and function of telomere via a telomere lengthening substitution mechanism (5). Mutant p53 protein (p53N236S) lost the function of inducing cell cycle inhibition and apoptosis, thereby obtaining the functions of promoting tumor formation and metastasis (6). Caspase-3, also known as cysteine protease 32, is a type of apoptosis-related gene that was closely associated with tumor occurrence and development. It constitutes the core position of the apoptotic cascade reaction pathway and is also known as death protease (7). The bcl-2 protein is the encoding product of bcl- 2 proto-oncogene. It is located in the mitochondria, endoplasmic reticulum, and continuous perinuclear membrane and may prevent the release of cytochrome $c$ from the mitochondrion to cytoplasm, thereby further inhibiting cell apoptosis (8).

In the present study, p53 N236S (p53S) gene knockout mice were examined and tumor formation effects were shown to be relatively stable (9). By establishing ovarian cancer models, aspirin of different concentrations was used for intervention. Subsequently, the intervention results of different groups were 
compared and possible molecular mechanisms were determined to provide a new treatment modality for ovarian cancer.

\section{Materials and methods}

Animals and reagents. In total, 28 male mice with stable p53S, an age range of 4-6 weeks and weight of 20-25 g, were purchased from the Kunming University of Science and Technology (Yunnan, China). The animals were fed at a room temperature of $22-24^{\circ} \mathrm{C}$, with humidity of $50-55 \%$, and a $12 \mathrm{~h}$ light/dark cycle was followed. The animals were allowed to adapt to the new environment for 1-2 weeks. Operations were carried out in a laminar airflow clean room. The human SKOV3 ovarian cancer cell strain was donated by the key laboratory of Dongfang Hospital (Beijing, China) and cultured in Dulbecco's modified Eagle's medium (DMEM), as well as high glucose medium containing $10 \%$ fetal bovine serum, and $100 \mathrm{U} / \mathrm{ml}$ penicillin and $100 \mathrm{U} / \mathrm{ml}$ streptomycin. Cell morphology and growth was observed under an inverted microscope (Olympus, Tokyo, Japan), after which the cells were passaged and the medium was changed every 2-3 days.

Aspirin (Sigma-Aldrich, St. Louis, MO, USA) was dissolved into $10 \mathrm{~mol} / \mathrm{l}$ sodium hydroxide solution with $\mathrm{pH}$ 7.0. Aspirin stock solution $(50 \mathrm{mmol} / \mathrm{l})$ was prepared in phosphate-buffered saline (PBS) and filter sterilized by $0.22 \mu \mathrm{mol} / 1$ prior to storing at $4^{\circ} \mathrm{C}$. Before use, the solution was diluted to different concentrations, i.e., 1,2 and $3 \mathrm{mmol} / \mathrm{l}$ by serum-free medium. PBS was purchased from Solarbio (Beijing, China), dimethyl sulfoxide (DMSO) was purchase from Sigma-Aldrich, and the Cell Counting Kit-8 (CCK-8) was purchased from Dojindo Molecular Technologies, Inc (Kumamoto, Japan). The two-step general immunohistochemical detection kit was purchased from Dako (Shanghai China), and 3,3'-diaminobenzidine (DAB) developing solution and $0.01 \mathrm{M}$ citrate buffer solution were purchased from Gene Tech Biotechnology Co., Ltd. (Shanghai, China). Forma Series II $\mathrm{CO}_{2}$ incubator was the product of Thermo Fisher Scientifie (Waltham, MA, USA); The XL-90 over-speed low temperature centrifuge was purchased from Beijing BJ-Genetool Co. Ltd. (Beijing, China); the DNM-9602 microplate reader was purchased from Beijing Perlong Medical Instrument Ltd. (Beijing, China); the adjustable pipette was purchased from Eppendorf AG (Hamburg, Germany); RM2125 type tissue sectioner was purchased from Leica (Mannheim, Germany); and 8122214 type optical microscope was purchased from Olympus (Tokyo, Japan).

Establishment of subdermal human SKOV3 ovarian cancer cell transplantation model. Skin from the back of mice left forelimb was selected and $4 \%$ sodium sulfide solution was injected. SKOV3 cells growing in the logarithmic phase were selected and washed twice with PBS to adjust cell concentration to $10 \times 10^{7} / \mathrm{ml}$. The tumor cells were injected in the animals. The aspirin solution of different concentration was used with $1 \mathrm{ml}$ syringe, for intraperitoneal injection once every two days for period of 3 weeks.

Grouping method and observation index. The mice were randomly divided into different concentration groups (mmol/l), i.e., 0, 1, 2 and 3, and were fed on normal diet and drinking water. Two mice from each group were sacrificed by cervical dislocation after 1,2 and 3 weeks. The tumor tissue was dissected for histological observation, as described elsewhere (10). The tumor volume and maximum diameter (a) and minimum diameter (b) of the tumor body were measured by vernier caliper. The tumor volume was calculated using the formula $\mathrm{V}=1 / 2 \times \mathrm{a} \mathrm{x} \mathrm{b}^{2}$, and the proliferation inhibition rate was determined by the CCK- 8 method. Caspase- 3 and bcl- 2 protein expression was determined using the immunohistochemical two-step method.

Procedures of CCK- 8 detection. The cells were collected and centrifuged at 2,000 $\mathrm{x}$ g for $3 \mathrm{~min}$. The pellet was washed with PBS, and $50 \mu$ l of cell suspension was taken to estimate cell concentration. The cell concentration was adjusted to $1 \times 10^{5} /$ $\mathrm{ml}$, and the cell suspension was inoculated into 96 -well plates, with each well containing $200 \mu \mathrm{l}$. Each group was cultured at $37^{\circ} \mathrm{C}$ for $48 \mathrm{~h}$, then $20 \mu \mathrm{l}$ of $10 \% \mathrm{CCK}-8$ solution was added into each pore plate by pipette. The culture was continued for $2 \mathrm{~h}$ in the incubator, the supernatant was removed and $200 \mu \mathrm{l}$ of DMSO was suspended. The culture was fully oscillated and dissolved to measure the absorbance (A) at $490 \mathrm{~nm}$ using a microplate reader (Shanghai Shenggong Technology Co. Ltd., Shanghai, China) within 30 min. Subsequently, the cell proliferation inhibition rate (IR) was calculated as: IR $=(1-\mathrm{A}$ of experiment group $>A$ of control group) $\mathrm{x} 100 \%$.

Immunohistochemical two-step detection. After dewaxing and hydration, the sections were washed with PBS three times for 3 min each. The sections were then placed in $0.01 \mathrm{M}$ citrate buffer, to retrieve the antigen for $2.5 \mathrm{~min}$ and then cooled $37^{\circ} \mathrm{C}$. The sections were washed again three times with PBS for $3 \mathrm{~min}$. Subsequently, $50 \mu \mathrm{l}$ of $3 \%$ hydrogen dioxide solution was added to each section and incubated at room temperature for $10 \mathrm{~min}$, followed by washing with PBS as mentioned earlier. Subsequently, $50 \mu \mathrm{l}$ of $3 \%$ primary antibody (1:100 rabbit anti-human caspase-3 antibody and 1:150 bcl-2 antibody) was added to each section and incubated at $37^{\circ} \mathrm{C}$ for $1 \mathrm{~h}$, followed by PBS washing. Fresh DAB solution (50 $\mu \mathrm{l})$ was added to observe the slides for 5 min under a microscope (Olympus). The solution turned the sections brown, after which the sections were counterstained with hematoxylin dye. The sections were then washed with distilled water sealed with neutral gum, and incubated in xylene (Fangzheng Chemical Co., Ltd., Chengdu,China). For interpretation of results, sepia, claybank or flavescent granule in cytoplasm indicated positive staining. Five visual fields were selected under a x400 magnified visual field, to calculate the positive rate and staining intensity. The positive rate of staining scoring was: 0 point, $0-5 \%$; 1 point, $6-25 \%$; 2 points, $26-50 \%$; and 3 points, 51-100\%; and scoring of staining intensity: 0 point, uncolored; 1 point, partially weak coloring; 2 points, weak to medium coloring; and 3 points, strong coloring.

The score value was calculated as: (sum of product of positive rate scoring and staining intensity scoring of each visual field) $/ 5$; range, $0-9$ points.

Statistical processing. SPSS 19.0 software (IBM, Armonk, NY, USA) was applied to for statistical analysis. Quantitative data were presented as mean \pm standard deviation. The variance 
Table I. Comparison of tumor volume $\left(\mathrm{cm}^{3}\right)$

\begin{tabular}{lcccc}
\hline Aspirin group & 0 week & 1 week & 2 weeks & 3 weeks \\
\hline $0 \mathrm{mmol} / 1$ & $3.2 \pm 0.3$ & $3.4 \pm 0.5$ & $3.5 \pm 0.6$ & $3.6 \pm 0.5$ \\
1 & $3.3 \pm 0.4$ & $3.2 \pm 0.6$ & $3.2 \pm 0.5$ & $3.1 \pm 0.6$ \\
2 & $3.2 \pm 0.2$ & $3.1 \pm 0.5$ & $3.0 \pm 0.6$ & $3.0 \pm 0.7$ \\
3 & $3.3 \pm 0.3$ & $2.7 \pm 0.6$ & $2.5 \pm 0.6$ & $2.3 \pm 0.8$ \\
F & 0.624 & 3.564 & 3.928 & 4.326 \\
P-value & 0.732 & 0.042 & 0.039 & 0.036 \\
\hline
\end{tabular}

Table II. Comparison of proliferation inhibition rate (\%).

\begin{tabular}{lcccc}
\hline Aspirin group & 0 week & 1 week & 2 weeks & 3 weeks \\
\hline $0 \mathrm{mmol} / \mathrm{l}$ & $0.22 \pm 0.03$ & $0.23 \pm 0.04$ & $0.24 \pm 0.03$ & $0.23 \pm 0.05$ \\
1 & $0.24 \pm 0.03$ & $9.25 \pm 1.25$ & $9.63 \pm 1.36$ & $9.55 \pm 1.53$ \\
2 & $0.28 \pm 0.02$ & $18.62 \pm 4.28$ & $19.27 \pm 4.32$ & $20.33 \pm 5.16$ \\
3 & $0.25 \pm 0.04$ & $27.73 \pm 5.36$ & $28.54 \pm 5.93$ & $30.32 \pm 4.78$ \\
$\mathrm{~F}$ & 0.854 & 4.128 & 4.329 & 4.502 \\
P-value & 0.329 & 0.034 & 0.027 & 0.025 \\
\hline
\end{tabular}

Table III. Comparison of caspase-3 and bcl-2 protein expression.

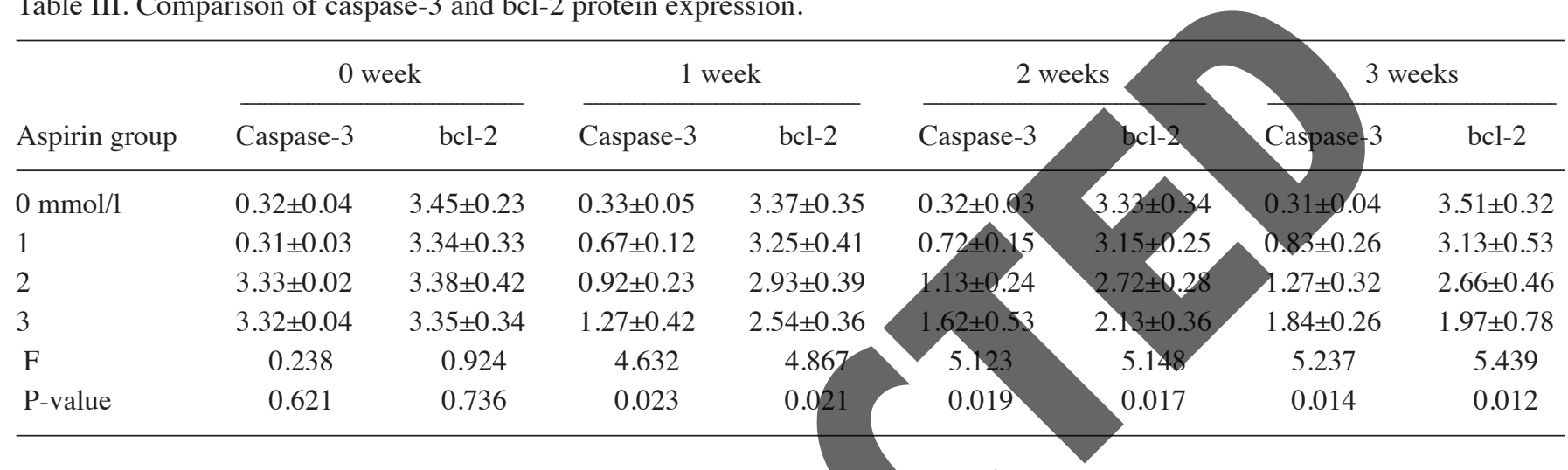

analysis and repeated measurement data test were used in aspirin treatment, came to the conclusion that aspirin was group comparisons, while enumeration data were presented by able to reduce the risks of ovarian cancer (11). Findings percentage (\%). The $\chi^{2}$ test was applied for group comparisons and $\mathrm{P}<0.05$ was considered to be statistically significant. of a previous study have showed that aspirin inhibits the

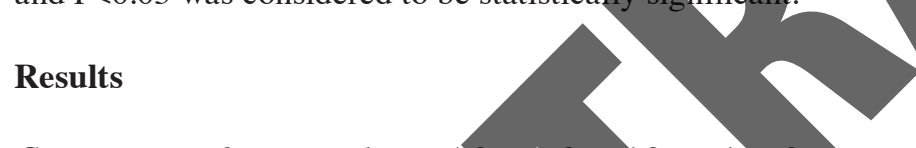

Comparison of tumor volume. After 1,2 and 3 weeks of treatment with aspirin, the tumor volume of the $3 \mathrm{mmol} / \mathrm{l}$ aspirin group was significantly smaller than that of the other groups $(\mathrm{P}<0.05)$. The higher concentration of aspirins significantly reduced the tumor size $(\mathrm{P}<0.05)$ (Table $\mathrm{I})$.

Comparison of proliferation inhibition rate. After 1, 2 and 3 weeks of aspirin treatment, the cell proliferation inhibition rate of the 3 mmolyl aspirin group was significantly larger than that of the other groups $(\mathrm{P}<0.05)$. The higher concentrations of aspirin significantly enhanced the cell proliferation inhibition rate $(\mathrm{P}<0.05)$ (Table II).

Comparison of caspase-3 and bcl-2 protein expression. After 1,2 and 3 weeks of treatment with aspirin, the relative expression of caspase- 3 protein with the $3 \mathrm{mmol} / \mathrm{l}$ aspirin group was significantly improved. The expression level of bcl-2 protein was significantly reduced, and higher aspirin concentrations significantly enhanced the caspase- 3 protein expression and reduced the bcl-2 protein expression $(\mathrm{P}<0.05)$ (Table III).

\section{Discussion}

Schildkraut et al after comparing and analyzing 585 ovarian cancer patients and 627 healthy individuals under 5-year proliferation and induces the apoptosis of ovarian cancer cells, and suppresses the expression of OVCAR-3 ovarian cancer cells by obstructing HER-2/neu receptor (12). Thus, inhibition of the metastasis of ovarian cancer by affecting metastasis-related genes, and its nitro derivative NCX-4016 may inhibit the proliferation of cisplatin-resistant ovarian cancer cells through obvious cytotoxic effects, thereby treating with recurrent ovarian cancer (13). In the recovery phase after epithelial ovarian cancer surgery, there is a possible risk of arterial thrombus. To improve the patient prognosis, Salman and Ayhan suggested treatment of the patients by conventional dose (75-100 $\mathrm{mg}$ /day) of aspirin until the end of chemotherapy (14). The results of their study showed that conventional dose (75-100 mg/day) of aspirin treatment after surgery significantly prevented the formation of thrombus, enhancing the cytotoxic effects of chemotherapeutics on microlesions, and improving the patient prognosis.

Although an increasing number of studies have suggested that aspirin inhibits the recurrence and metastasis of malignant tumors, such as colorectal, liver cancer, and ovarian cancer, and increases the sensitivity of chemotherapy (15), in clinic, there remains a lack of consensus regarding the specific mechanism of action and the optimal concentration (16). In the present study, ovarian cancer models were established, and aspirin of different concentrations was used for intervention. The intervention results of the different groups were compared and a possible molecular mechanism was determined. The results showed that 1, 2 and 3 weeks after aspirin treatment, the tumor volume with treatment of $3 \mathrm{mmol} / \mathrm{l}$ aspirin was 
significantly smaller than that of the other groups. The higher concentrations of aspirin significantly reduced the tumor size, but enhanced the cell proliferation inhibition rate compared to the other groups. The relative expression level of caspase-3 protein with $3 \mathrm{mmol} / \mathrm{l}$ aspirin was significantly improved. The expression of bcl-2 was significantly reduced, and higher concentrations of aspirin led to significant inhibition of cell proliferation. Additionally, caspase-3 expression was increased whereas that of bcl-2 was reduced. Although data from this study are promising, the small sample size included in the present study was a limitation of this study.

In conclusion, aspirin can inhibit the growth of ovarian cancer of p53S rats, possibly as it was able to upregulate the expression of caspase- 3 protein and downregulate the expression of Bcl-2 protein.

\section{References}

1. Tarim E, Bal N, Kilicdag E, Kayaselcuk F, Bağis T and Kuscu E: Effects of aspirin on placenta and perinatal outcomes in patients with poor obstetric history. Arch Gynecol Obstet 274: 209-214, 2006.

2. Middleton FA, Ignacio C, Camargo M, Hicks S and Mooney S: Effects of developmental ethanol exposures in wildtype and p53-null mice on transcriptional and epigenetic regulation of DNA damage repair, cell cycle, cell fate and cell death processes. Int J Dev Neurosci 47: 124-125, 2015.

3. Park YJ, Kim EK, Bae JY, Moon S and Kim J: Human telomerase reverse transcriptase (hTERT) promotes cancer invasion by modulating cathepsin D via early growth response (EGR)-1. Cancer Lett 370: 222-231, 2015.

4. Hansen GL, Gaudernack G, Brunsvig PF, Cvancarova M and Kyte JA: Immunological factors influencing clinical outcome in lung cancer patients after telomerase peptide vaccination. Cancer Immunol Immunother: Oct 26, 2015 (Epub ahead of print).
5. Shi M, Zheng J, Liu C, Tan G, Qing Z, Yang S, Yang J, Tan Y and Yang R: SERS assay of telomerase activity at single-cell level and colon cancer tissues via quadratic signal amplification. Biosens Bioelectron 77: 673-680, 2016.

6. Moorchung N, Vasudevan B, Dinesh Kumar S and Muralidhar A: Expression of apoptosis regulating proteins p53 and bcl-2 in psoriasis. Indian J Pathol Microbiol 58: 423-426, 2015.

7. Croci DO, Cogno IS, Vittar NB, Salvatierra E, Trajtenberg F, Podhajcer OL, Osinaga E, Rabinovich GA and Rivarola VA: Silencing survivin gene expression promotes apoptosis of human breast cancer cells through a caspase-independent pathway. J Cell Biochem 105: 381-390, 2008.

8. Gordeev SA, Bykova TV, Zubova SG, Aksenov ND and Pospelova TV: Antiapoptotic gene bcl-2 prevents cellular senescence program reactivation induced by histone deacetylase inhibitor sodium butyrate in E1A and cHa-ras transformed rat fibroblasts. Tsitologiia 57: 135-143, 2015 (In Russian).

9. Muller PA and Vousden KH: Mutant p53 in cancer: new functions and therapeutic opportunities. Cancer Cell 25: 304-317, 2014.

10. Padda RS, Gkouvatsos K, Guido M, Mui J, Vali H and Pantopoulos K: A high-fat diet modulates iron metabolism but does not promote liver fibrosis in hemochromatotic $\mathrm{Hjv}^{-/}$mice. Am J Physiol Gastrointest Liver Physiol 308: G251-G261, 2015.

11. Schildkraut JM, Moorman PG, Halabi S, Calingaert B, Marks JR and Berchuck A: Analgesic drug use and risk of ovarian cancer. Epidemiology 17: 104-107, 2006.

12. Drake JG and Becker JL: Aspirintinduced inhibition of ovariantumor cell growth. Obstet Gynecol 100: 677-682, 2002.

13. Andrews P, Zhao X, Allen J, Li F and Chang M: A comparison of the effectiveness of selected non-steroidal anti-inflammatory drugs and their derivatives against cancer cells in vitro. Cancer Chemother Pharmacol 11: 203-214, 2007.

. Salman MC and Ayhan A: Use of anti-thrombotic agents during chemotherapy for epithelial ovarian cancer. Med Hypotheses 66 : $1179-1181,2006$

15. McMenamin ÚC, Cardwell CR, Hughes CM and Murray LM: Low-dose aspirin and survival from lung cancer: a population-based cohort study. BMC Cancer 15: 911, 2015.

6. Kim B, Park SJ, Hong SP, Cheon JH, Kim WH and Kim TI: The effect of prediagnostic aspirin use on the prognosis of stage III colorectal cancer. Int J Clin Exp Med 8: 13435-13445, 2015.

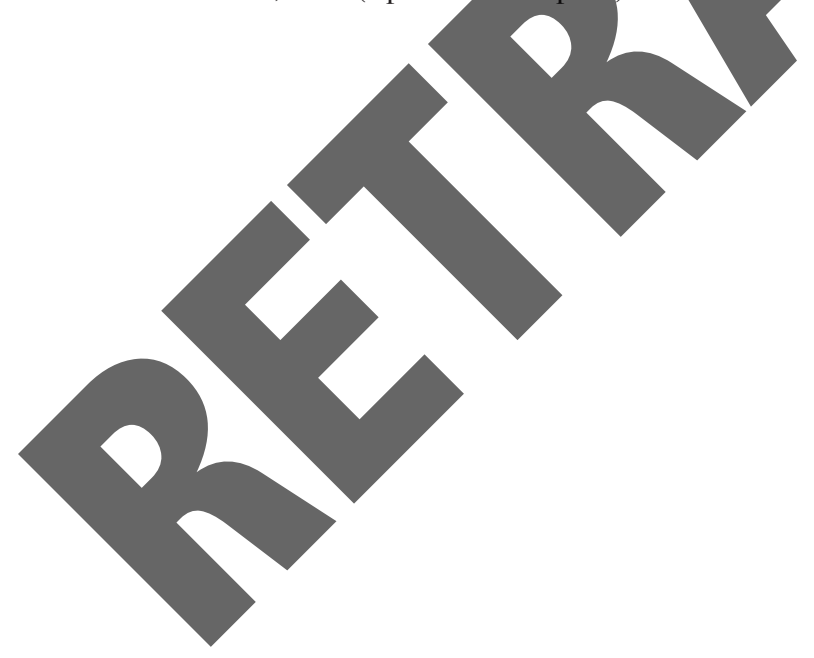

